\title{
Properties of some time dependent probability failure functions in reliability theory
}

\author{
V. Zardasht ${ }^{1}$ \\ M. Asadi \\ P. Zeephongsekul ${ }^{3}$
}

(Received 19 June 2010; revised 23 July 2010)

\begin{abstract}
We consider systems having many independent components connected in a parallel or series configuration with non-identical failure distributions. A time dependent measure is proposed which evaluate the probability that a failure of the system occurred at a specified component under the condition that the system has failed by some time. We proved several properties of this measure for parallel and serial systems.
\end{abstract}

\section{Contents}

1 Introduction

C513

2 Parallel systems

C514

2.1 Parallel system with two components . . . . . . . . . .

\section{Series systems}

http://anziamj . austms . org. au/ojs/index.php/ANZIAMJ/article/view/2418 gives this article, (c) Austral. Mathematical Soc. 2010. Published July 30, 2010. ISSN 1446-8735. (Print two pages per sheet of paper.) 


\section{Introduction}

Several failure models have been proposed and failure probabilities evaluated in reliability research by people such as Beichelt and Fischer [2], Nakagawa [7], Sheu and Griffith [10], and Cha and Mi [3]. We are interested in the following failure probability. Consider a system with $n$ components. Let $X_{i}$ be the lifetime of component $i, i=1,2, \ldots, n$, and $T$ is the lifetime of the system. If the system is connected in series, then $T=\min _{1 \leqslant i \leqslant n} X_{i}$ and, if it is a parallel connection, then $T=\max _{1 \leqslant i \leqslant n} X_{i}$. Cha and Mi [3] considered the following probability function and studied its properties

$$
p_{j}(t)=\operatorname{Pr}\left(X_{j}=T \mid T=t\right) .
$$

That is, the probability that the failure of the system is caused by the $j$ th component given that it fails at time $t$. However, in real applications we do not usually observe the exact time when the system fails but rather whether the system has already failed prior to $t$, that is instead of the conditioned event in (1) being $T=t$, it is more appropriate to consider $T \leqslant t$. Motivated by this fact, the objective of this study is the probability function

$$
\mathrm{L}_{n}^{j}(\mathrm{t})=\operatorname{Pr}\left(\mathrm{X}_{\mathrm{j}}=\mathrm{T} \mid \mathrm{T} \leqslant \mathrm{t}\right) .
$$

When the system is parallel, this probability can be used to obtain information on the probability of failure time of the components of the system. In a series system, it determines the failed component of the system. The expressions, after we let $t \rightarrow \infty$ in (1) and (2), correspond to the importance measures of components in a coherent system discussed by Barlow and Proschan [1].

We consider various properties of $L_{n}^{j}(t)$ for parallel system and series system in Section 2 and Section 3 respectively. But first we overview some preliminary 
concepts related to ageing and partial orderings between random variables which will be referred to later on. The two ageing concepts defined for a random variable $X$ with distribution function $F(x)$ and density $f(x)$ are the reversed hazard rate (RHR)

$$
r_{X}(t)=\lim _{\delta \rightarrow 0} \frac{\operatorname{Pr}(t-\delta<X<t \mid X \leqslant t)}{\delta}=\frac{f(t)}{F(t)},
$$

and mean past lifetime (MPL)

$$
m_{X}(t)=E(t-X \mid X \leqslant t)=\frac{\int_{0}^{t} F(x) d x}{F(t)},
$$

provided $F(t)>0$. The RHR gives the failure rate of the component just before time $t$, whereas the MPL corresponds to the mean time elapsed since the failure of $X$ given that $X \leqslant t$.

The symbol $X \mid A$ denotes the random variable $X$ conditional on an event $A$. The symbol $\overline{\mathrm{F}}=1-\mathrm{F}$ refers to the reliability function. Also, increasing means non-decreasing and decreasing will mean non-increasing. We next give some related partial ordering concepts which will be referred to later [9].

- A random variable $Y$ is said to be smaller than $X$ in the usual stochastic order (denoted by $Y \leqslant$ st $X$ ) if $\bar{G}(x) \leqslant \bar{F}(x)$ for all $x$.

- $X$ is said to be smaller than $Y$ in the RHR order (denoted by $X \leqslant_{\text {rh }} Y$ ) if $r_{X}(t) \leqslant r_{Y}(t)$, for all $t>0$.

- $X$ is said to be less than the random variable $Y$ in the MPL ordering (written as $X \leqslant{ }_{m p l} Y$ ) if $m_{X}(t) \geqslant m_{Y}(t)$ for all $t>0$.

\section{Parallel systems}

A system is said to have a parallel structure if it will only operate if at least one of its components operate. We consider a parallel system with $n$ components. 
Assume that $X_{i}$, the lifetime of $i$ th component, $i=1,2, \ldots, n$, has distribution function $F_{i}$, reliability function $\bar{F}_{i}$, RHR $r_{i}$ and MPL $m_{i}$. Furthermore, let $X_{k: n}$, $k=1,2, \ldots, n$, denote the $k$ th ordered lifetime ( $k$ th order statistic) of the components. Suppose that at the time of monitoring the system, the operator realizes that the system has already failed. That is, the operator knows that $X_{n: n} \leqslant t$, where $t$ is the time the system is monitored. The probability that this was caused by component $j$ is

$$
\begin{aligned}
& \mathrm{L}_{\mathrm{kn}}^{\mathrm{j}}(\mathrm{t})=\operatorname{Pr}\left(X_{k: n}=X_{j} \mid X_{n: n} \leqslant \mathrm{t}\right) \\
& =\frac{1}{\prod_{i=1}^{n} F_{i}(\mathrm{t})} \sum_{\pi} \operatorname{Pr}\left(X_{\pi_{1}}<\cdots<X_{\pi_{k-1}}<X_{j}<X_{\pi_{k+1}}<\cdots<X_{\pi_{n}} \leqslant \mathrm{t}\right),
\end{aligned}
$$

where $\pi_{1}, \ldots, \pi_{k-1}, \pi_{k+1}, \ldots, \pi_{n}$ is a permutation of $\{1, \ldots, j-1, j+1, \ldots, n\}$. For example, in the case $n=3, k=1, j=2$,

$$
\mathrm{L}_{13}^{2}(\mathrm{t})=\frac{1}{\prod_{i=1}^{3} \mathrm{~F}_{\mathrm{i}}(\mathrm{t})}\left[\operatorname{Pr}\left(\mathrm{X}_{2}<\mathrm{X}_{1}<\mathrm{X}_{3} \leqslant \mathrm{t}\right)+\operatorname{Pr}\left(\mathrm{X}_{2}<\mathrm{X}_{3}<\mathrm{X}_{1} \leqslant \mathrm{t}\right)\right] .
$$

We concentrate on a special case which is of particular interest to engineers and system designers. This case arises when we take $k=n$, corresponding to $T=X_{n: n}$, the lifetime of the system. Here, $L_{n n}^{j}(t)=L_{n}^{j}(t)$ defined in (2), which is the probability that the lifetime of the system is equal to that of the jth component of the system, under the condition that system has failed by time $t$. First note that, for $j=1,2, \ldots, n$, we obtain from (5) that

$$
\mathrm{L}_{n}^{j}(t)=\operatorname{Pr}\left(X_{n: n}=X_{j} \mid X_{n: n} \leqslant t\right)=\frac{1}{\prod_{k=1}^{n} F_{k}(t)} \int_{0}^{t} \prod_{\substack{i=1 \\ i \neq j}}^{n} F_{i}\left(x_{j}\right) d F_{j}\left(x_{j}\right) .
$$

The proportional RHRs model is a dual model of the well-known proportional hazards model (introduced by Cox [4]). The concept of proportional RHRS, which have applications in survival analysis and life testing, is introduced by O'Neill [8] and studied by others [6, e.g.]. Two random variables $\mathrm{X}$ and $\mathrm{Y}$, 
with distribution functions F and G, are said to have proportional Reversed Hazards Rates (RHRS) if there exists $\mathrm{c}>0$ such that

$$
F(t)=[G(t)]^{c}, \quad t>0 .
$$

If the RHRS of $X$ and $Y$ exist, that is the distribution functions are absolutely continuous, then (7) is equivalent to

$$
r_{X}(t)=c r_{Y}(t)
$$

for any $t>0$. For further properties and applications of this model see Gupta and Gupta [5] and references therein.

Theorem 1 For any $\mathrm{j}=1,2, \ldots, \mathrm{n}, \mathrm{L}_{\mathrm{n}}^{\mathrm{j}}(\mathrm{t})$ is independent of time if and only if $\max _{i \neq j}\left\{X_{i}\right\}$ and $X_{j}$ have proportional RHRS.

Proof. Denote by $r_{\max _{i \neq j}\left\{X_{i}\right\}}(t)$ and $r_{X_{j}}(t)$ the RHRS of $\max _{i \neq j}\left\{X_{i}\right\}$ and $X_{j}$, $j=1,2, \ldots, n$, respectively. Then (7) implies

$$
\prod_{\substack{i=1 \\ i \neq j}}^{n} F_{i}(t)=\left(F_{j}(t)\right)^{c}
$$

for some $c>0$. Using (6), we readily obtain $L_{n}^{j}(t)=1 /(c+1)$ which is independent of time. Conversely, assume that $L_{n}^{j}(t)=b$ where $b$ is a constant such that $0<b<1$. Differentiating both sides of (6) with respect to $t$ gives

$$
r_{X_{j}}(t)=b\left(r_{\max _{i \neq j}\left\{X_{i}\right\}}(t)+r_{X_{j}}(t)\right)
$$

or

$$
r_{X_{j}}(t)=\frac{b}{1-b} r_{\max _{i \neq j}\left\{X_{i}\right\}}(t),
$$

therefore $\max _{i \neq j}\left(X_{i}\right)$ and $X_{j}$, have proportional RHRS. In particular, if $b=$ $1 / 2$, then $\max _{i \neq j}\left(X_{i}\right)$ and $X_{j}$ have identical distribution.

The next theorem shows that if the RHRS of two components of the system are ordered, then their corresponding $\mathrm{L}(\mathrm{t})$ functions are also ordered. 
Theorem 2 Let $r_{j_{i}}(t), i=1,2$, denote the RHR of the $j_{i}$ th component of the system, $j_{i}=1,2, \ldots, n$, If $r_{j_{1}}(t) \leqslant r_{j_{2}}(t)$ for any $\mathrm{t}>0$, then

$$
\mathrm{L}_{n}^{\mathrm{j}_{1}}(\mathrm{t}) \leqslant \mathrm{L}_{n}^{\mathrm{j}_{2}}(\mathrm{t}) .
$$

Proof: Using (6),

$$
\mathrm{L}_{n}^{j_{2}}(t)-\mathrm{L}_{n}^{j_{1}}(t)=\frac{1}{\prod_{k=1}^{n} F_{k}(t)} \int_{0}^{t} \prod_{i=1}^{n} F_{i}(x)\left[r_{j_{2}}(x)-r_{j_{1}}(x)\right] d x \geqslant 0
$$

under the assumption of the theorem and this completes the proof.

Note that when the system is parallel, the probability function defined in (1) is equal to

$$
p_{j}(t)=\operatorname{Pr}\left(X_{j}=X_{n: n} \mid X_{n: n}=t\right) .
$$

Cha and Mi [3] showed that $p_{j}(t)$ can be represented in terms of the RHRS of the components as

$$
p_{j}(t)=\frac{r_{j}(t)}{\sum_{i=1}^{n} r_{i}(t)} .
$$

On differentiating the right hand side of (6), one easily shows that $L_{n}^{j}(t)$ is increasing (decreasing ) in $t$ if and only if

$$
\mathrm{L}_{n}^{\mathrm{j}}(\mathrm{t}) \leqslant(\geqslant) \mathrm{p}_{j}(\mathrm{t})
$$

\subsection{Parallel system with two components}

We consider the special case where the system consists of two components. Let $X$ and $Y$ be the lifetime of the components having distribution functions $F$ and $G$, and survival functions $\overline{\mathrm{F}}$ and $\overline{\mathrm{G}}$, respectively. Let

$$
\mathrm{L}(\mathrm{t})=\operatorname{Pr}(\max \{X, Y\}=X \mid \max \{X, Y\} \leqslant t),
$$


which is the probability that the component with lifetime $\mathrm{X}$ causes the failure of the system, given that the lifetime of the system is less than or equal to $t$. We have

$$
L(t)=\frac{\int_{0}^{t} G(x) d F(x)}{F(t) G(t)}=\frac{\int_{0}^{t} G(x) f(x) d x}{\int_{0}^{t} F(x) g(x) d x+\int_{0}^{t} G(x) f(x) d x},
$$

provided that $F(t)>0$ and $G(t)>0$.

The following theorem shows that the behaviour of $\mathrm{L}(\mathrm{t})$ is closely related to the behaviour of $\rho(t)=r_{X}(t) / r_{Y}(t)$, where $r_{X}(t)$ and $r_{Y}(t)$ are the RHRS of $X$ and $Y$ respectively.

Theorem 3 Let $\mathcal{S}_{\mathrm{L}}=\{\mathrm{t}: \mathrm{F}(\mathrm{t})>0, \mathrm{G}(\mathrm{t})>0\}$ and assume that both $\mathrm{F}(\mathrm{t})$ and $\mathrm{G}(\mathrm{t})$ are absolutely continuous with respect to $\mathrm{t}$.

1. For all $\mathrm{t} \in \mathcal{S}_{\mathrm{L}}$, if $\rho(\mathrm{t})$ is increasing (decreasing) function of $\mathrm{t}$, then $\mathrm{L}(\mathrm{t})$ is an increasing (decreasing) function of $\mathrm{t}$.

2. For all $\mathrm{t} \in \mathcal{S}_{\mathrm{L}}, \mathrm{L}(\mathrm{t})$ is independent of $\mathrm{t}$ if and only if $\rho(\mathrm{t})$ is independent of $\mathrm{t}$.

3. $\mathrm{L}(\mathrm{t})$ has a peak (valley) at $\mathrm{t}^{*}>\mathrm{t}_{0}$ if $\rho(\mathrm{t})$ has a peak (valley) at $\mathrm{t}_{0}$.

\section{Proof:}

1. Note that

$$
L(t)=\frac{\varphi(t)}{1+\varphi(t)}, \quad \text { where } \quad \varphi(t)=\frac{\int_{0}^{t} G(x) f(x) d x}{\int_{0}^{t} F(x) g(x) d x} .
$$

Since the function $x /(1+x)$ is strictly increasing in $x>0$, it follows that the monotonicity of $L(t)$ is the same as that of $\varphi(t)$. On the other hand,

$$
\varphi(t)=\frac{\int_{0}^{t} F(x) G(x) r_{X}(x) d x}{\int_{0}^{t} F(x) G(x) r_{Y}(x) d x}=\frac{\int_{0}^{t} w(x) f_{m}(x) d x}{\int_{0}^{t}(1-w(x)) f_{m}(x) d x}
$$


where $w(x)=\rho(x) /(1+\rho(x))$ and $f_{m}(x)=f(x) G(x)+g(x) F(x)$ is the density of $F_{\max \{X, Y\}}(x)=F(x) G(x)$. Clearly the monotonicity of $\rho(x)$ determines that of $w(x)$. Thus it is enough to show that the monotonicity of $\mathcal{w}(x)$ determines that of $\varphi(x)$. We obtain

$$
\varphi^{\prime}(t)=\frac{f_{m}(t) \int_{0}^{t}[w(t)-w(x)] f_{m}(x) d x}{\left.\left[\int_{0}^{t}(1-w(x)) f_{m}(x) d x\right)\right]^{2}}
$$

which show that $\varphi^{\prime}(t) \geqslant(\leqslant) 0$ depending upon whether $w(t)$ is increasing (decreasing) in $t$. This concludes the proof of part 1 of the theorem.

2. Part 2 of the theorem follows from Theorem 1 adapted to the case $n=2$ and the definition of $\rho(t)$.

3. To show that part 3 of the theorem is true, assume that $w(x)$ has a peak (valley) at $t_{0}$. Then from (11), for all $t$ such that $t \leqslant t_{0}$ we have $\varphi^{\prime}(t)>(<) 0$. On the other hand, for all $t$ such that $t>t_{0}$, since $w(t)$ is decreasing (increasing), it can be shown that the expression $\int_{0}^{t} f_{m}(x)[w(t)-w(x)] d x$ in the numerator of (11) is decreasing (increasing) in $t$. This implies that there exists a value of $t^{*}>t_{0}$ where $\varphi^{\prime}(t)$ changes sign; that is, $\varphi(t)$, and hence $\mathrm{L}(\mathrm{t})$, has a peak (valley) at $t^{*}$. This completes the proof of part 3 .

Example 4 Let $\mathrm{X}$ and $\mathrm{Y}$ be distributed as $\mathrm{F}$ and $\mathrm{G}$, where

$$
F(t)=1-\frac{1}{1+4 t^{2}}, \quad t>0,
$$

and

$$
\mathrm{G}(\mathrm{t})=1-\mathrm{e}^{-3 \mathrm{t}^{2}}, \quad \mathrm{t}>0 .
$$

Figure 1 shows the plot of $\rho(t)$ and $L(t)$ corresponding to $F$ and $G$. The plots show that when $\rho(t)$ is bathtub shape then the corresponding $L(t)$ also has 

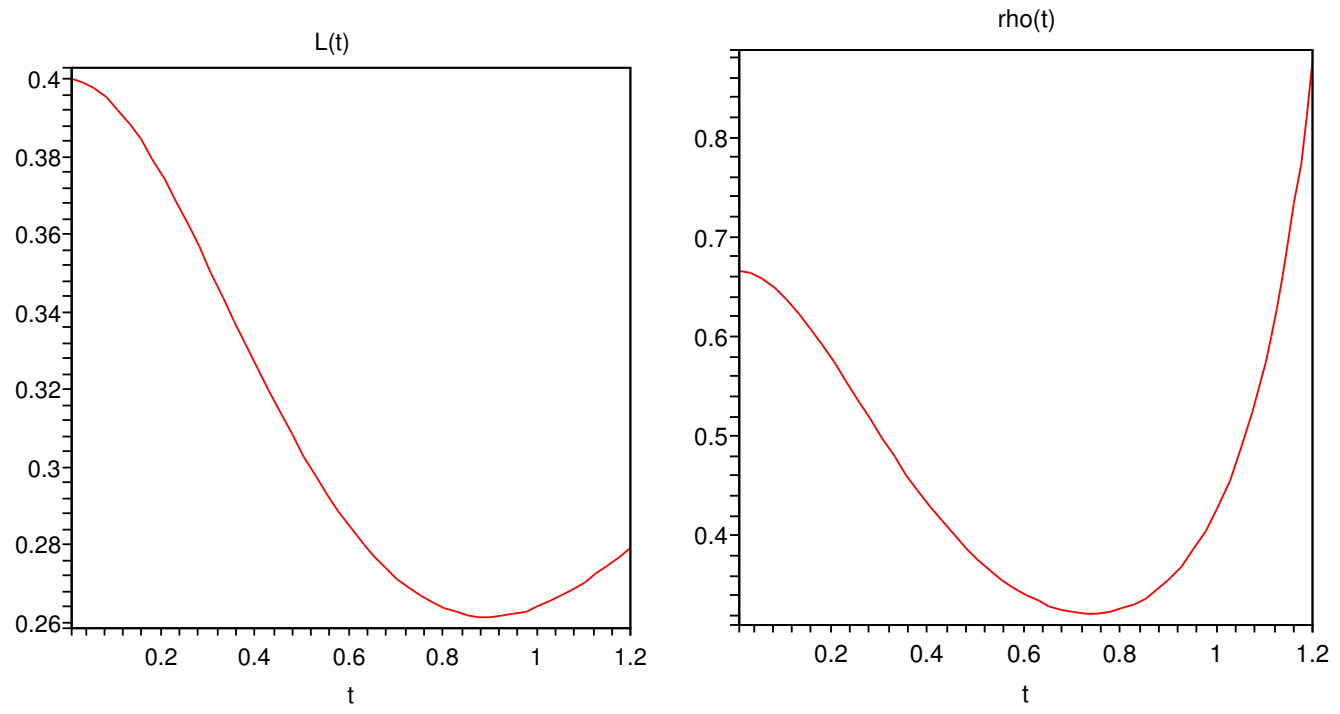

Figure 1: The plot of $\mathrm{L}(\mathrm{t})$, at left, and the plot of $\rho(\mathrm{t})$, at right, in Example 4.

a bathtub shape. Note also the valley of $L(t)$ is located to the right of that of $\rho(t)$ as predicted by part 3 of Theorem 3

When $X$ and $Y$ have the same distribution, (10) implies that $L(t)=1 / 2$. On the other hand, differentiating (10) with respect to $t$ gives

$$
L^{\prime}(t)=\left[r_{X}(t)+r_{Y}(t)\right][p(t)-L(t)] .
$$

From (12), when $L(t)=1 / 2$, we have $r_{X}(t)=r_{Y}(t)$. Therefore, $X$ and $Y$ have identical distributions. Motivated by this, we introduce the concept " $Y$ is stronger than X" in the following definition.

Definition 5 Let $\mathrm{X}$ and $\mathrm{Y}$ be two non-negative continuous random variables representing the lifetimes of two components. The component with lifetime $\mathrm{Y}$ is said to be stronger than the component with lifetime $\mathrm{X}$ (denoted by $\mathrm{X} \leqslant_{s r} \mathrm{Y}$ ) if, for all $\mathrm{t}>0, \mathrm{~L}(\mathrm{t}) \leqslant 1 / 2$.

Theorem 6 If $\mathrm{X} \leqslant_{r h} \mathrm{Y}$, then $\mathrm{X} \leqslant_{s r} \mathrm{Y}$. 
Proof: From equation (10) we see that $X \leqslant_{s r} \mathrm{Y}$ if and only if

$$
\int_{0}^{t} G(x) f(x) d x \leqslant \int_{0}^{t} F(x) g(x) d x .
$$

The assumption that $X \leqslant$ rh $Y$, implies $f(x) G(x) \leqslant g(x) F(x)$ for all $x>0$, therefore (13) holds and this completes the proof.

The following example indicates that the converse of above theorem is not true.

Example 7 Let $X$ and $Y$ be distributed exponentially with mean 1 and 0.5 respectively. Then

$$
L(t)=\frac{3\left(1-e^{-2 t}\right)-2\left(1-e^{-3 t}\right)}{3\left(1-e^{-t}\right)\left(1-e^{-2 t}\right)} \leqslant \frac{1}{2} .
$$

On the other hand, $r_{X}(0.05)=19.46$ and $r_{Y}(0.05)=18.98$. Hence there exists at least one $t$ such that $r_{X}(t)>r_{Y}(t)$ thus violating $X \leqslant_{r h} Y$.

The following theorem gives a necessary and sufficient condition for $X \leqslant_{\text {sr }} Y$. Theorem $8 \quad X \leqslant_{s r} Y$ if and only if $\mathrm{F}(\mathrm{X}) \leqslant_{m p l} \mathrm{~F}(\mathrm{Y})$.

Proof: Let $Z=F(Y)$ and $W=F(X)$ and note that $W$ is uniformly distributed over $(0,1)$. Denote by $m_{Z}(t)$ the MPL function of $Z$. Then it follows after some manipulations that

$$
\mathrm{L}(\mathrm{t})=\frac{\mathrm{m}_{\mathrm{Z}}(\mathrm{F}(\mathrm{t}))}{\mathrm{F}(\mathrm{t})} .
$$

Now, $\mathrm{X} \leqslant$ sr $\mathrm{Y}$ implies that $\mathrm{L}(\mathrm{t}) \leqslant 1 / 2$ for $\mathrm{t}>0$ which in turn implies $m_{Z}(F(t)) / F(t) \leqslant 1 / 2$ or equivalently, $m_{Z}(u) \leqslant u / 2$ for $0<u<1$. Since $\mathfrak{m}_{W}(\mathfrak{u})=\mathfrak{u} / 2$, it follows that $F(X) \leqslant_{\text {mpl }} F(Y)$. The converse follows by reversing the steps of the proof. 


\section{Series systems}

A system consisting of $\mathrm{n}$ components is said to have a series structure if it operates as long as every component in the system is operating. If $X_{i}$, $i=1,2, \ldots, n$, is the lifetime of component $i$, then the lifetime of the series system is $T=\min _{1 \leqslant i \leqslant n} X_{i}$. In this case $L_{n}^{j}(t)$, the probability that component $j$ caused the failure of the system, given that the failure time of the system is less than or equal $t$, is

$$
L_{n}^{j}(t)=\operatorname{Pr}\left(X_{1: n}=X_{j} \mid X_{1: n} \leqslant t\right)=\frac{1}{1-\prod_{i=1}^{n} \bar{F}_{i}(t)} \int_{0}^{t} \prod_{i=1}^{n} \bar{F}_{i}(x) h_{j}(x) d x,
$$

where $h_{j}(x)=f_{j}(x) / \bar{F}_{j}(x)$ is the hazard function of $X_{j}$.

Some of the results in the parallel components case hold here as well and we do not repeat them. For example, the following theorem is analogous to Theorem 2 and the proof is thereby omitted.

Theorem 9 Let $\mathrm{h}_{\mathrm{j}_{\mathrm{i}}}(\mathrm{t}), \boldsymbol{i}=1,2$, denote the hazard rate of the $\mathrm{j}_{\mathrm{i}}$ th component of the system, $\mathrm{j}_{\mathrm{i}}=1,2, \ldots, \mathrm{n}$, If $\mathrm{h}_{\mathrm{j}_{1}}(\mathrm{t}) \leqslant \mathrm{h}_{\mathrm{j}_{2}}(\mathrm{t})$, for all $\mathrm{t}>0$, then

$$
L_{n}^{j_{1}}(t) \leqslant L_{n}^{j_{2}}(t) \text {. }
$$

Consider the special case with two components and let $X$ and $Y$ be the lifetimes of the components having distribution functions $F$ and $G$, and survival functions $\overline{\mathrm{F}}=1-\mathrm{F}$ and $\overline{\mathrm{G}}=1-\mathrm{G}$, respectively. Let

$$
\mathrm{L}(\mathrm{t})=\operatorname{Pr}(\min \{X, Y\}=X \mid \min \{X, Y\} \leqslant t) ;
$$

that is, $\mathrm{L}(\mathrm{t})$ is the probability that the component with lifetime $\mathrm{X}$ causes the failure of the system, given that the lifetime of the system is less than or equal to $t$. Then

$$
L(t)=\frac{\int_{0}^{t} \bar{G}(x) d F(x)}{\int_{0}^{t} \bar{G}(x) d F(x)+\int_{0}^{t} \bar{F}(x) d G(x)},
$$


provided that $\bar{F}(t)>0$ and $\bar{G}(t)>0$. Similar to Theorem 3 one can show that the behaviour of $L(t)$ is closely related to the behaviour of $\rho(t)=h_{X}(t) / h_{Y}(t)$, where $h_{X}(t)$ and $h_{Y}(t)$ denote the hazard rate function of $X$ and $Y$ respectively.

\section{Conclusion}

We considered an $n$ component system connected in a parallel or series configuration which can fail prior to time $t$ and studied in detail the probability that the failure of the system is caused by its $j$ th component, $j=1,2, \ldots, n$. When the system is parallel, this probability can be used to obtain information on the probability of failure time of the components of the system. In a series system it determines the failed component of the system. This probability is an extension of the probability considered by Cha and Mi [3] and may be used as an alternative in various areas of reliability applications such as in general failure models.

In the case of a parallel system with two components, we were able to relate various stochastic ordering relations such as reversed hazard rates to the probability that one of the components caused the system failure. Graphs also display the relationships.

Future research on this topic will extend the case of two components consider here for parallel systems to more than two components. It will also integrate other stochastic order relationships discussed by Shaked and Shantikumar [9] to the particular probability of system failure considered in this article.

Acknowledgment The authors thank the Editor, an associate Editor and two anonymous referees, for their valuable and constructive comments, which improved the presentation of the article. The first author is grateful to the office of Graduate Studies of the University of Isfahan for their support. This work is supported in part by the School of Mathematical and Geospatial Sciences, RMIT university. 


\section{References}

[1] R. E. Barlow and F. Proschan. Importance of System Components and Fault Tree Events. Stochastic Processes and their Applications, 3:153-173, 1975. C513

[2] F. Beichelt and K. Fischer. General failure model applied to preventive maintenance policies. IEEE Trans. Rel., 29:39-41, 1980. C513

[3] J. H. Cha and J. Mi. Some Probability Functions in Reliability and Their Applications. Naval Research Logistics., 54:128-135, 2007. doi:10.1002/nav.20192 C513, C517, C523

[4] D. R. Cox. Regression models and life-tables (with discussion). J. R. Stat. Soc., B34:187-208, 1972. C515

[5] R. C. Gupta and R. D. Gupta. Proportional reversed hazard rate model and its applications. J. Statist. Plann. Inference, 137:3525-3536, 2007. doi:10.1016/j.jspi.2007.03.029 C516

[6] R. C. Gupta, R. D. Gupta and P. L. Gupta. Modeling failure time data by Lehman alternatives. Comm. Statist. Theory Methods, 27:887-904, 1998. doi:10.1080/03610929808832134 C515

[7] T. Nakagava. Generalized models for determining optimal number of minimal repairs before replacement. J. Operat. Res. Soc. Japan, 24:325-357, 1981. C513

[8] T. J. O'Neill. The inverse Proportional Hazards Model. Stat. and Prob. Letters, 12:125-129, 1991. doi:10.1016/0167-7152(91)90055-V C515

[9] M. Shaked, and J. G. Shanthikumar. Stochastic Orders., Springer, 2007. C514, C523

[10] S. H. Sheu, and W. S. Griffith. Optimal number of minimal repairs before replacement of a system subject to shocks. Naval Res. Logist., 43:319-333, 1996. 
doi:10.1002/(SICI)1520-6750(199604)43:3ز319::AID-NAV1_3.0.CO;2-C C513

\section{Author addresses}

1. V. Zardasht, Department of Statistics, University of Isfahan, Isfahan, IRAN.

mailto:v.zardasht@sci.ui.ac.ir

2. M. Asadi, Department of Statistics, University of Isfahan, Isfahan, IRAN.

3. P. Zeephongsekul, School of Mathematical and Geospatial Sciences, RMIT University, Melbourne, Victoria, Australia. 